\title{
Preoperative CA-125 Values as a Predictive Factor for the Postoperative Outcome in Primary Serous Ovarian Cancer
}

\author{
MUSTAFA ZELAL MUALLEM ${ }^{1 *}$, ASYA PARASHKEVOVA ${ }^{2 *}$, JUMANA ALMUHEIMID $^{1}$, \\ ROLF RICHTER $^{1}$, YASSER DIAB ${ }^{3}$, ELENA IOANA BRAICU ${ }^{1,2}$ and JALID SEHOULI ${ }^{1}$ \\ ${ }^{1}$ Department of Gynecology, and ${ }^{2}$ Ovarian Cancer Tumor Bank, \\ Virchow Campus Clinic, Charité Medical University, Berlin, Germany; \\ ${ }^{3}$ Department of Gynecology, Portland District Hospital, Victoria, Australia
}

\begin{abstract}
Background/Aim: The purpose of the study was to examine the preoperative CA-125 values as a predictive factor for postoperative outcome in primary serous ovarian cancer (POC) for complete tumor resection (CTR) and evaluate the preoperative CA-125 levels with other vital clinical dynamics such as ascites, lymph node involvement, diffuse peritoneal carcinomatosis, grading and staging. Patients and Methods: A cohort of 277 POC-patients aged 18-75 years, who had undergone primary cytoreductive surgery at the Department of Gynecology \& Oncological Surgery, Charité, Campus Virchow Klinikum (CVK) between 2000 und 2009 was analyzed in correlation with the preoperative CA-125 values. Results: The median preoperative CA-125 value in high-grade serous $P O C$ patients was $636 \mathrm{U} / \mathrm{ml}$ (204- $2312 \mathrm{U} / \mathrm{ml}$ ) compared to $284 \mathrm{U} / \mathrm{ml}$ (148.5-1,378 U/ml) in low-grade serous POC patients $(p=0.016)$. For the survival analyses both the cut-off values 252 and $475 \mathrm{U} / \mathrm{ml}$, with highest sum from sensitivity $(79.1 \%$ and $65.9 \%$, respectively) and specificity $(41.9 \%$ and $55.1 \%$, respectively), were used to compare the relationship between preoperative CA-125 levels and (CTR), progression-free (PFS) and overall survival (OS). There was no significant difference between PFS and OS in three different groups of patients (preoperative CA-125 levels $<252 \mathrm{U} / \mathrm{ml}, \mathrm{CA} 125$ levels between 252-475 U/ml and $>475 \mathrm{U} / \mathrm{ml}$ ). Conclusion: Preoperative CA125 is a poor, but statistically significant predictive factor for CTR after PCS. Preoperative CA-125 can predict neither the progression-free nor overall survival for POC patients.
\end{abstract}

*These Authors contributed equally to this study.

Correspondence to: Dr. med. Dr. (syr.) Mustafa Zelal Muallem, Charité University Medicine Berlin, Campus Virchow-Klinikum, Augustenburger Platz 1, 13353 Berlin, Germany. Tel: +49 30450664373, Fax: +49 30450564900, e-mail: MustafaZelal.Muallem@charite.de

Key Words: Ovarian cancer, CA-125, primary cytoreductive surgery, complete tumor resection.
Epithelial ovarian cancer (EOC) is the seventh most common cancer worldwide, with 238,700 new cases and 151,900 deaths in 2012 (1). It remains the major cause of death from gynecologic malignancies and represents the fifth leading cause of all cancer-deaths among women (2).

CA-125 is a repeating peptide epitope of mucin (MUC) 16 (3-4), which promotes cancer cell proliferation and inhibits anti-cancer immune responses (5-6). This and published evidence pointing to an altered expression of the protein in ovarian cancers (7) have spurred the interest on CA-125 as a biomarker for serous ovarian cancer (8). Numerous research studies have investigated the role of preoperative CA-125 value as predictor for postoperative outcome: some of them reviewed only patients with epithelial ovarian cancer $(2,9)$, or the predictive value of preoperative CA-125 together with preoperative computed tomography scan (CT) in patient groups with advanced ovarian, fallopian tube and peritoneal cancer disease (10). However, the predictive value of CA-125 levels has been inconclusive (11), therefore research has also focused on another tumor marker like Human epididymal protein 4 (HE4) in serum plasma in order to predict the surgical outcome in Primary ovarian cancer (POC) patients (12).

The objective aim of our retrospective study was to examine the predictive value of preoperative CA-125 for optimal cytoreduction and postoperative overall survival after primary cytoreductive surgery (PCS) and evaluate the preoperative CA-125 levels with other vital clinical dynamics such as ascites, lymph node involvement, diffuse peritoneal carcinomatosis, grading and staging.

\section{Materials and Methods}

The database from the Tumor Bank Ovarian Cancer (www.tocnetwork.de) was utilized to identify patients with the required specific case history. This database, essentially, a prospective documentation tool includes clinical data, disease history, tumor spread, presence of ascites, and presence and location of residual tumor mass intra-operatively obtained through an interview with the 
surgeon immediately post-operatively. The study had in place the requisite informed consent before collection of the clinical data and the ethics committee approval from Charite local Ethics Committee (EK207/2003). Cancer staging in the study was based on The International Federation of Gynecology and Obstetrics (FIGO) classification prior to 2014 to match the database (13). The optimal surgery was defined as no macroscopic tumor residual after PCS.

We included all serous POC-patients aged 18-75 years, which underwent PCS at the department of Gynecology with oncological surgery, Charité, Campus Virchow Klinikum (CVK) between 2000 and 2009. The design had an exclusion criterion of patients with non-epithelial ovarian cancer, other histologies than serous, patients with no preoperative CA-125 evaluations, women who had undergone interval surgery, and those who were treated for another malignant disease in the last 5 years of the study period.

The statistical analysis has been performed at the Charite Medical University Berlin. All analyses have been performed by IBM SPSS Statistics 22.0 (SPSS, Chicago, IL, USA).

Descriptive statistic tests were used to characterize the patient cohort and Kolmogorov Simirnov test to characterize the distribution of preoperative CA-125 levels.

With Receiving operating characteristic (ROC) analysis sensitivity and specificity were calculated to define the optimal cutoff value of the preoperative CA-125 levels for predicting Complete tumor resection (CTR).

The correlation between preoperative CA-125 levels and clinical factors such as age, ascites, FIGO stage and CTR was investigated using non-parametric univariate tests such as Kendall-Tau b, Spearman Rho, Man-Whitney and Kruskal Wallis test.

Median and 95\% confidence intervals (95\%CI) of Progression free survival (PFS) and Overall survival (OS) were estimated according to the Kaplan-Meier method. PFS was defined as the length of time between the end of the last chemotherapy cycle to the occurrence of the relapse. OS was determined as the length of time between the date of first diagnosis and the date of death or end of follow-up. Log-rank test statistics for analysis of the equality of survival distribution were performed. Statistical significance was defined by $p<0.05$ and two-sided tests were applied.

\section{Results}

The patient characteristics are presented in Table I. A total of 227 patients in a POC cohort were investigated and CA-125 measured before the primary cytoreductive surgery in all patients. The median age of the cohort at the first diagnosis was 59 years. One in four patients was younger than 50 or older than 65 years. A percentage of $96.9 \%$ of them had an advanced recurrent POC (FIGO III-IV). A high-grade serous POC had been diagnosed in $64.5 \%$ of cases, with $81.5 \%$ of the patients with ascites $(44.1 \%$ with less than $500 \mathrm{ml}$ and $37.4 \%$ with more than $500 \mathrm{ml}$ ). A percentage of $59.9 \%$ of the women had been operated with no macroscopic tumor residual and $85.5 \%$ of the operated women had undergone some type of lymph node dissection. A percentage of $55.5 \%$ of them have had pelvic and/or paraaortal lymph node metastasis at the time of operation. The median follow-up time was 44.4 months (range $=0.1-158$ months). The main CA-125 value was 1991.9; the median was $552 \mathrm{U} / \mathrm{ml}$ (the interquartile range=IQR: 164-
Table I. Characteristics of 227 primary serous ovarian cancer patients underwent primary cytoreductive surgery in Charité Berlin.

\begin{tabular}{lc}
\hline Characteristics & $\begin{array}{c}\text { All patients } \\
\mathrm{n}=227(\%)\end{array}$ \\
\hline Age at first diagnosis [years] & Median $59(21-92)$ \\
CA-125 & 552 U/mL $(11-38387)$ \\
FIGO classification & $7(3.1 \%)$ \\
II & $178(78.4 \%)$ \\
III & $42(18.5 \%)$ \\
IV & \\
Grading & $80(35.2 \%)$ \\
$1-2$ & $147(64.8 \%)$ \\
3 & \\
Ascites & $42(18.5 \%)$ \\
No ascites & $100(44.1 \%)$ \\
$<500$ ml & $85(37.4 \%)$ \\
Residual tumour & \\
No residual & $136(59.9 \%)$ \\
$<10$ mm & $64(28.2 \%)$ \\
$\geq 10$ mm & $27(11.9 \%)$ \\
Lymph nodes status & \\
N1 & $68(30 \%)$ \\
Nx & $126(55.5 \%)$ \\
\hline
\end{tabular}

$2,004 \mathrm{U} / \mathrm{ml})$. The median preoperative CA-125 value in highgrade serous POC patients was $636 \mathrm{U} / \mathrm{ml}$ (range=204-2,312 $\mathrm{U} / \mathrm{ml}$ ) compared to $284 \mathrm{U} / \mathrm{ml}$ (range $=148.5-1,378 \mathrm{U} / \mathrm{ml})$ in low-grade serous POC patients $(p=0.016)$. KolmogorovSmirnov Test does not display normal distribution of CA-125 in the cohort (Figure 1-A) whereas, the logarithmic conversed CA-125 values illustrate a normal, symmetric distribution curve (Figure 1-B).

The correlation between preoperative CA-125 levels and the outcomes of surgery (CTR). Since, the Area Under Curve (AUC) has a small surface, to predict the prognostic value of preoperative CA-125; it was prudent to set a cut-off value of preoperative CA-125, which predicts the CTR. Thus, the estimated optimal CA-125 preoperative cut-off value in our study was $252 \mathrm{U} / \mathrm{ml}$ and $475 \mathrm{U} / \mathrm{ml}$ with highest sum from sensitivity (79.1\% and $65.9 \%$, respectively) and specificity (41.9\% and $55.1 \%$, respectively) (together, namely 121 ), so that we could predict the chance for CTR for $79.1 \%$ of patients, who had CA-125 less than $252 \mathrm{U} / \mathrm{ml}$ before the primary cytoreductive surgery and for $65.9 \%$ of patients with preoperative CA-125 of less than $475 \mathrm{U} / \mathrm{ml}$.

The correlation between preoperative CA-125 levels and PFS or OS. For the survival analyses both the cut off values 252 and $475 \mathrm{U} / \mathrm{ml}$ were used to compare the relationship 
A

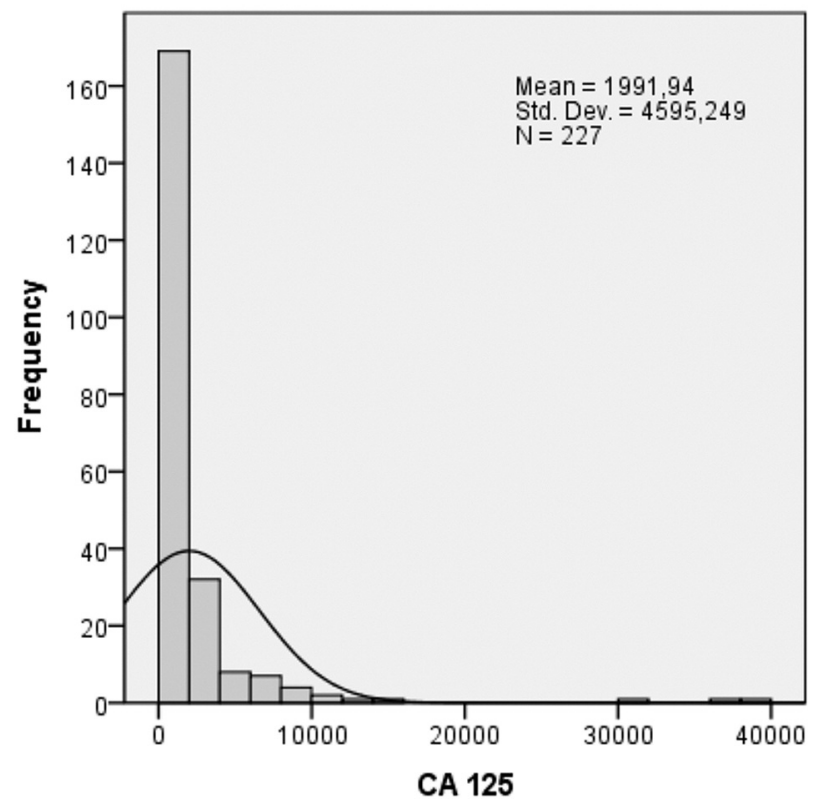

B

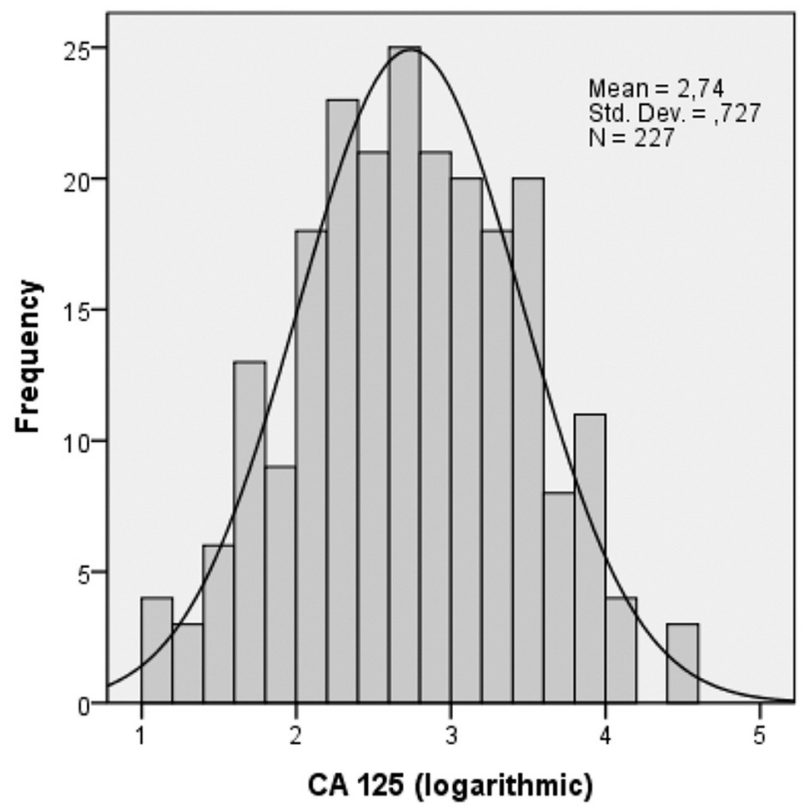

Figure 1. The distribution of CA-125 values in our collective. A: Kolmogorov-Smirnov Test with normal distribution. B: Logarithmic conversed CA125 values with symmetric curve of distribution.
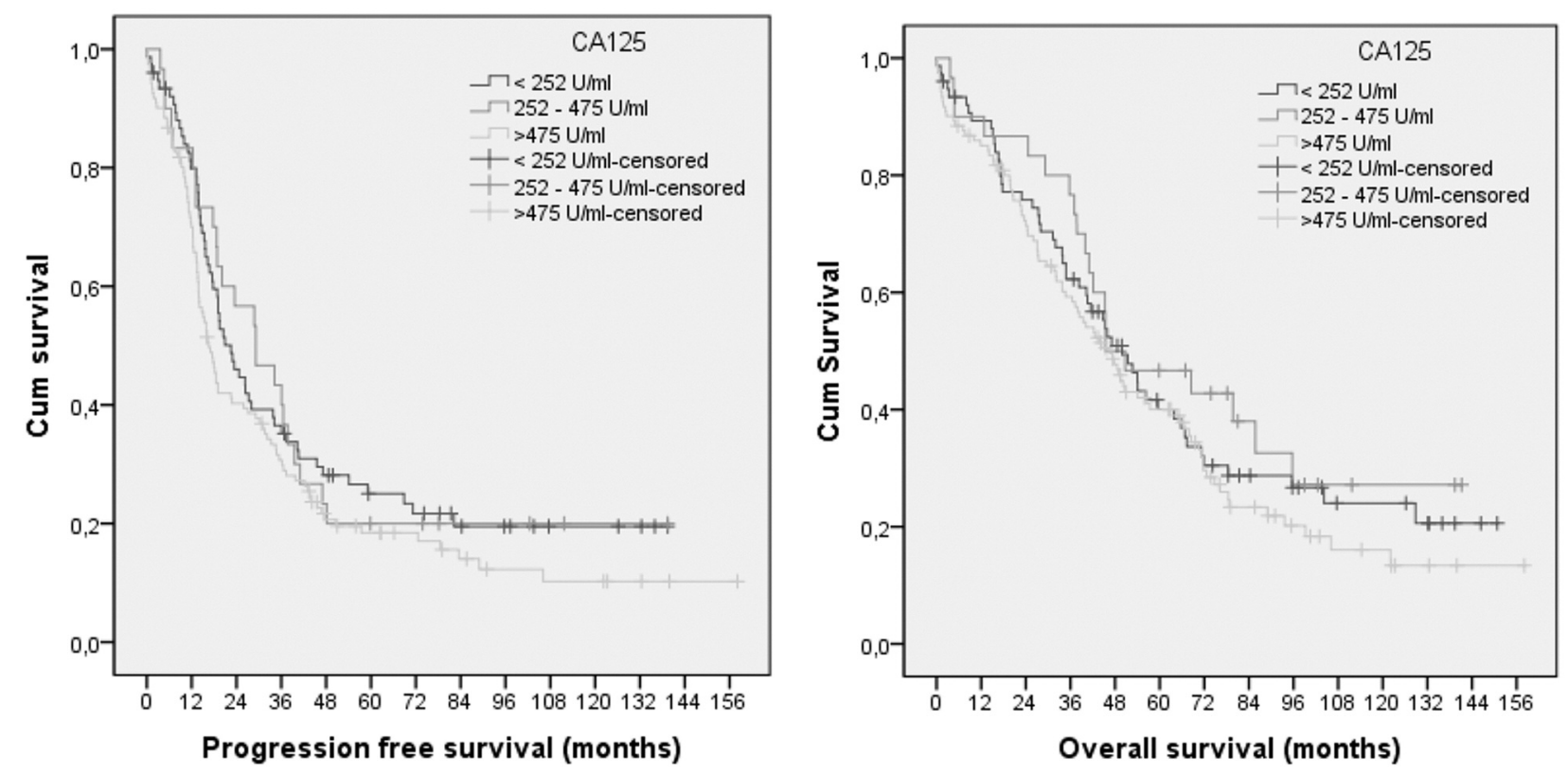

Figure 2. Progression-free and overall survival in the 3 groups of primary serous ovarian cancer patients according to preoperative CA-125 values.

between preoperative CA-125 levels and PFS or OS. There was no significant difference between PFS and OS in three different groups of patients (preoperative CA-125 levels $<252 \mathrm{U} / \mathrm{ml}, \mathrm{CA}-125$ levels between $252-475 \mathrm{U} / \mathrm{ml}$ and $>475 \mathrm{U} / \mathrm{ml}$ ) (Figure 2).
The correlation between preoperative CA-125 values and the important clinical prognostic factors. Univariate analysis of the conventional key clinical prognostic factors for POC and its correlation to preoperative CA-125 showed no correlation between preoperative CA-125 and the patient's age. However, 
a correlation was observed with advanced POC (FIGO III-IV) in comparison with early staged POC (FIGO I-II); with ascites $>500 \mathrm{ml}$ in comparison with no ascites or ascites $<500 \mathrm{ml}$; with high-grade serous POC in comparison with low-grade serous POC and with CTR in comparison with residual tumor ( $p$-value $=0.579,0.008,0.000,0.014$ and 0.001 respectively). Interestingly, lymph node status was an important factor, which correlated significantly $(p=0.029)$ with preoperative CA-125. The Kruskal-Wallis test for lymph node metastasis showed a significant correlation in patients with lymph nodes metastasis (N1) in comparison to those without any lymph node metastasis (N0), $(p=0.015)$ and in patients who did not undergo lymph nodes dissection (when CTR is not possible) (Nx) in comparison with those without any lymph node metastasis (N0), $(p=0.039)$.

\section{Discussion}

One of the statistically significant cut-off values using ROC analysis of preoperative CA-125 levels in the study has been $475 \mathrm{U} / \mathrm{ml}$ with best sensitivity $(65.9 \%)$ and specificity $(55.1 \%)$. The value matches with other similar studies that have reported that an optimal cytoreduction (defined here as with residual tumor less than $1 \mathrm{~cm}$ ) could be predicted only in $50 \%$ of cases with FIGO III-staged epithelial POC using a cut off value of preoperative CA-125 of $500 \mathrm{U} / \mathrm{ml}$. The study has also elucidated that the chance of an optimal cytoreduction decreases with higher values of preoperative CA-125 (14).

These results fall in tandem with studies of Mury et al. (15) that have analyzed the prognostic and predictive values of CA-125 serum concentrations before and after surgery as well as their correlation with clinicopathological variables in a collective of 231 patients with POC (with different histological subtypes). Despite of correlation with some surgical results, they could not determine a preoperative cutoff value for prediction of the surgical result. A prognostic relevance was only observed for postoperative CA-125 in stage III/IV patients.

The study also falls in line with the study of Memarzadeh et al. (9) in 99 patients with stage IIIC and IV epithelial POC aiming to test the utility of preoperative CA-125 to predict optimal primary tumor cytoreduction (defined here as largest volume of residual disease $<1 \mathrm{~cm}$ in maximal dimension). Optimal cytoreduction was achieved in $73 \%$ of patients with a mean CA-125 level of $569 \mathrm{U} / \mathrm{ml}$, while among patients with suboptimal cytoreduction the mean CA-125 level was $1520 \mathrm{U} / \mathrm{ml}(p<0.007)$. With a CA-125 level of $912 \mathrm{U} / \mathrm{ml}$ identified as the optimal cut-point to distinguish the two groups, the sensitivity of the test in predicting optimal cytoreduction has been just $58 \%$ (in tandem with $79.1 \%$ and $65.9 \%$, respectively for our both cutoff values 252 and 475 $\mathrm{U} / \mathrm{ml}$ in present study) and the specificity $54 \%$ leading us to the conclusion that CA-125 level is a weak positive and negative predictor of optimal cytoreductive surgery in patients with advanced epithelial ovarian cancer.

Similar study by Chi et al. (16) have also concluded that preoperative CA-125 does not predict the primary cytoreductive outcome of patients with advanced ovarian, tubal, or peritoneal carcinoma. However, with a preoperative CA-125 >500 U/ml, extensive upper abdominal procedures were necessary in $50 \%$ of cases to achieve residual disease $\leq 1 \mathrm{~cm}$. Interestingly, CTR was achieved only in $25 \%$ of their collective.

Harter et al. (17) reviewed prognostic factors for CTR after first- and second-line ovarian cancer. They could not find sufficient predictive markers (including the preoperative CA-125) for CTR.

In the European multi-centric project "Ovarian Cancer: Diagnosis of a silent killer" (OVCAD) (12), 275 consecutive patients with POC were enrolled and analyzed. The results showed that the combination of HE4 and CA-125 expression in plasma might predict the surgical outcome in EOC which may have a prognostic impact on PFS and OS. Plasma CA125 and the risk index (HE4 and CA-125) were independent predictive factors for surgical outcome $(p=0.001, \mathrm{OR}=3.37$, $95 \% \mathrm{CI}=1.61-7.06$ and $p<0.001, \mathrm{OR}=6.041,95 \% \mathrm{CI}=2.33-$ 15.65 , respectively).

Limiting factors of this study were the retrospective design, and the FIGO classifications provided were defined according to the old classification system. Nevertheless, our study has convincing advantages. The study cohort was restricted to patients with serous POC, thus only patients where preoperative CA-125 values assumed to be reflecting the peritoneal dissemination of cancer were included. In addition, the rather high number of patients in this collective, the high rate of CTR and the long median follow-up period add more value to our results.

In conclusion, preoperative CA-125 is poor, but statistically significant predictive factor for CTR after PCS. Preoperative CA-125 can predict neither the progression-free nor the overall survival for POC patients.

\section{Conflicts of Interest}

The Authors declare in financial or personal conflict of interest.

\section{References}

1 Torre LA, Bray F, Siegel RL, Ferlay J, Lortet-Tieulent J and Jemal A: Global Cancer Statistics, 2012. CA Cancer J Clin 65(2): 87-108, 2015.

2 Díaz-Padilla I, Razak AR, Minig L, Bernardini MQ and María Del Campo J: Prognostic and predictive value of CA-125 in the primary treatment of epithelial ovarian cancer: potentials and pitfalls. Clin Transl Oncol 14(1): 15-20, 2012.

3 O'Brien TJ, Beard JB, Underwood LJ, Dennis RA, Santin AD and York L: The CA 125 gene: an extracellular superstructure dominated by repeat sequences. Tumour Biol 22(6): 348-366, 2001. 
4 Yin BW and Lloyd KO: Molecular cloning of the ca125 ovarian cancer antigen identification as a new mucin, muc16. J Biol Chem 276(29): 27371-27375, 2001.

5 Bast RC Jr. and Spriggs DR: More than a biomarker: CA125 may contribute to ovarian cancer pathogenesis. Gynecol Oncol 121(3): 429-430, 2011.

6 Comamala M, Pinard M, Theriault C, Matte I, Albert A, Boivin M, Beaudin J, Piche A and Rancourt C: Down-regulation of cell surface CA125/MUC16 induces epithelial-to-mesenchymal transition and restores EGFR signalling in NIH:OVCAR3 ovarian carcinoma cells. Br J Cancer 104(6): 989-999, 2011.

7 Bafna S, Kaur S and Batra SK: Membrane-bound mucins: the mechanistic basis for alterations in the growth and survival of cancer cells. Oncogene 29(20): 2893-2904, 2010.

8 Suh KS, Park SW, Castro A, Patel H, Blake P, Liang M and Goy A: Ovarian cancer biomarkers for molecular biosensors and translational medicine. Expert Rev Mol Diagn 10(8): 1069-1083, 2010.

9 Memarzadeh S, Lee SB, Berek JS and Farias-Eisner R: CA 125 levels are weak predictor of optimal cytoreductive surgery in patients with advanced epithelial ovarian cancer. Int J Gynecol Cancer 13(2): 120-124, 2003.

10 Suidan RS, Ramirez PT, Sarasohn DM, Teitcher JB, Mironov S, Iyer RB, Zhou Q, Iasonos A, Paul H, Hosaka M, Aghajanian CA, Leitao MM Jr, Gardner GJ, Abu-Rustum NR, Sonoda Y, Levine DA, Hricak H and Chi DS: A multicenter prospective trial evaluating the ability of preoperative computed tomography scan and serum CA-125 to predict suboptimalcytoreduction at primary debulking surgery for advanced ovarian, fallopian tube, and peritoneal cancer. Gynecol Oncol 134(3): 455-461, 2014.

11 Moyer VA and U.S. Preventive Services Task Force: Screening for ovarian cancer: U.S. Preventive Services Task Force reaffirmation recommendation statement. Ann Intern Med 157(12): 900-904, 2012.
12 Braicu EI, Fotopoulou C, Van Gorp T, Richter R, Chekerov R, Hall C, Butz H, Castillo-Tong DC, Mahner S, Zeillinger R, Concin N, Vergote I and Sehouli J: Preoperative HE4 expression in plasma predicts surgical outcome in primary ovarian cancer patients. Results from the OVCAD study. Gynecol Oncol 128(2): 245-251, 2013.

13 Staging Announcement: FIGO Cancer Committee Gynecol Oncol 25: 383, 1986.

14 Chi DS, Venkatraman ES, Masson V and Hoskins WJ: The ability of preoperative serum CA-125 to predict optimal primary tumor cytoreduction in stage III epithelial ovarian carcinoma. Gynecol Oncol 77(2): 227-231, 2000.

15 Mury D, Woelber L, Jung S, Eulenburg C, Choschzick M, Witzel I, Schwarz J, Jaenicke F and Mahner S: Prognostic and predictive relevance of CA.125 at primary surgery of ovarian cancer. J Cancer Res Clin Oncol 137(7): 1131-1137, 2011.

16 Chi DS, Zivanovic O, Palayekar MJ, Eisenhauer EL, AbuRustum NR, Sonoda Y, Levine DA, Leitao MM, Brown CL and Barakat RR: A contemporary analysis of the ability of preoperative serum CA-125 to predict primary cytoreductive outcome in patients with advanced ovarian, tubal and peritoneal carcinoma. Gynecol Oncol 112(1): 6-10, 2009.

17 Harter P, Hilpert F, Mahner S, Kommoss S, Heitz F, Pfisterer J and du Bois A: Prognostic factors for complete debulking in first- and second-line ovarian cancer. Int J Gynecol Cancer 19(2): 14-17, 2009.

Received April 26, 2017

Revised May 3, 2017

Accepted May 8, 2017 